\title{
Estudo da simulação de um processo de produção de massa de rosca de uma padaria
}

\author{
Simulation study of a dough production process in a bakery \\ Estudio de simulación de un proceso de producción de masa de una panadería
}

Recebido: 15/10/2021 | Revisado: 19/10/2021 | Aceito: 20/10/2021 | Publicado: 22/10/2021

Pedro Ramalho Figueirêdo

ORCID: https://orcid.org/0000-0002-8989-2521 Universidade Federal dos Vales do Jequitinhonha e Mucuri, Brasil E-mail: figueiredo.pedro@ufvjm.edu.br

Lucas Portes Lacerda

ORCID: https://orcid.org/0000-0001-5558-2931 Universidade Federal dos Vales do Jequitinhonha e Mucuri, Brasil E-mail: lucas.portes@ufvjm.edu.br

Jaqueline Maria da Silva

ORCID: https://orcid.org/0000-0002-5713-1533

Universidade Federal dos Vales do Jequitinhonha e Mucuri, Brasil E-mail: jaqueline.silva@ufvjm.edu.br

Alexandre Faissal Brito

ORCID: https://orcid.org/0000-0002-6311-772X

Universidade Federal dos Vales do Jequitinhonha e Mucuri, Brasil E-mail: alexandre.faissal@ufvjm.edu.br

\begin{abstract}
Resumo
A simulação computacional é uma ferramenta com o potencial de gerar uma grande redução de custos operacionais, visto que é possível modelar um processo em ambiente controlável, testar modificações e analisar os resultados, sem necessariamente alterar a estrutura real do processo produtivo. Pequenas e médias empresas buscam novas vias de desenvolvimento com foco em novas fontes de vantagem competitiva, e a simulação computacional pode ser uma delas. Neste trabalho, buscou-se identificar os processos produtivos envolvidos na produção de uma padaria no município de Itambacuri/MG, escolher um desses processos e a partir do uso de simulação computacional com o software Arena, propor caso haja, melhorias e otimizações para a sua maximização produtiva. O produto escolhido como estudo foi a massa de rosca. Foram coletados dados referentes a cada processo de todos os produtos gerados pela massa. Para cada processo estocástico, são obtidos seus tempos de operação com o uso de um cronômetro em visita in loco. O modelo computacional construído foi verificado e validado com sucesso em comparação com dados do processo real. Através dos resultados obtidos pela simulação computacional foi possível identificar os gargalos no sistema e propor alternativas para a otimização do processo produtivo.
\end{abstract}

Palavras-chave: Modelagem computacional; Otimização; Padaria.

\begin{abstract}
Computer simulation is a tool with the potential to generate a large cost reduction for companies, since it is possible to model a process in a controllable environment, test modifications and analyze the results, without the necessity of changing the real production process. Small and medium-sized companies are looking for new development paths focusing on new sources of competitive advantage, and simulation may be one of them. In this work, we sought to identify the processes involved in the production of a bakery in the city of Itambacuri / MG (Brazil), to choose one of these processes and with the use of computer simulation with software Arena, propose improvements and optimizations for the production maximization. The product chosen for the study was the braided bread dough. Data was collected for each process of all products generated by the dough. For each stochastic process, its operating times are obtained with the use of a stopwatch during visit in loco. The simulation model built was verified and validated with success in comparison with real data. Through the results of the simulation, it was possible to identify bottlenecks in the system and to propose alternatives for the optimization of the production process.
\end{abstract}

Keywords: Computer simulation; Optimization; Bakery.

\section{Resumen}

La simulación por computadora es una herramienta con el potencial de generar una gran reducción en los costos operativos, ya que es posible modelar un proceso en un ambiente controlable, probar modificaciones y analizar los resultados, sin necesariamente cambiar la estructura real del proceso productivo. Las pequeñas y medianas empresas están buscando nuevas vías de desarrollo con un enfoque en nuevas fuentes de ventaja competitiva, y la simulación puede ser una de ellas. En este trabajo, se buscó identificar los procesos productivos involucrados en la producción de 
una panadería en el municipio de Itambacuri / MG, elegir uno de estos procesos y, a partir del uso de la simulación por computadora con el software Arena, proponer, en su caso, mejoras y optimizaciones para su máxima producción. El producto elegido como estudio fue la rosquilla. Se recogieron datos referentes a cada proceso de todos los productos generados por la masa. Para cada proceso estocástico, sus tiempos de funcionamiento se obtienen mediante un cronómetro in situ. El modelo computacional construido se verificó y validó con éxito con los datos del proceso real. A través de los resultados de la simulación, fue posible identificar los cuellos de botella en el sistema y proponer alternativas para la optimización del proceso productivo.

Palabras clave: Modelado computacional; Mejoramiento; Panadería.

\section{Introdução}

O crescente mercado competitivo e a consequente disputa pela ampliação da produtividade leva muitas empresas a buscarem novas formas de conquistarem mais espaço. A popularização dos computadores pessoais e da tecnologia de informação, em geral, proporcionou uma difusão de ferramentas de produtividade de maneira mais acessível. A evolução do marketing, evolução de processos produtivos, melhorias administrativas; tiveram uma contribuição importante no setor comercial e industrial do final do século XX para os dias de hoje.

A simulação computacional também é uma outra ferramenta que passou a fazer parte do arsenal de um bom gestor. A partir dela, e com um minucioso estudo de caso, é possível simular situações reais em um ambiente controlado, onde podem ser tomados riscos e observando os seus possíveis resultados. Tem um potencial muito grande de proporcionar a geração de ideias inovadoras para os gestores. Pode-se por exemplo alterar o processo produtivo de um determinado produto ou variar o número de funcionários operando em um dado momento e observar os impactos nos custos e produtividade (Kelton, 2002). E isso sem alterar o processo real de fato.

O ARENA é um dos softwares mais conhecidos na área de simulação computacional e é altamente difundido em empresas por todo o mundo. Com este tipo de software, pode-se simular processos reais, identificando e estudando cada etapa envolvida em um processo de produção para posterior análise de possíveis otimizações. Esta visão estratégica pode desencadear ganhos consideráveis em produção, lucro da empresa e melhorias no ambiente de trabalho.

Além de ser eficiente no seu funcionamento interno, o seu maior destaque está na sua acessibilidade e interface intuitiva. Seu visual permite que o fluxo de entidades seja observado claramente e pode ser facilmente modificado para melhor apresentação. A Gartner, empresa de consultoria tecnológica internacional, classifica a simulação computacional (mais especificamente o digital twin) como um dos grandes pilares da Indústria 4.0 (ou quarta revolução industrial) (Moura \& Nascimento, 2020).

Segundo a Leap Austrália (LEAP, 2020), esta emergente transformação foi desencadeada por tendências tecnológicas globais que permitem a convergência de sistemas físico-digitais, causando transformações massivas na maneira como os fabricantes criam, prestam serviços de manutenção e operam seus novos produtos inteligentes e conectados, envolvendo conceitos como big data, internet of things e machine learning.

Entretanto, para pequenas e médias empresas a situação muitas vezes é diferente. Elas geralmente não possuem um setor responsável pela avaliação e otimização de seus processos produtivos. Padarias são exemplos de pequenas e médias indústrias com cadeias produtivas que podem ser beneficiadas com a simulação computacional. Este setor de empreendimento vem buscando novas vias de desenvolvimento capazes de lidar com a complexidade crescente do ambiente de negócios e garantir acesso a novas fontes de vantagem competitiva.

O cenário competitivo dos setores comercial e industrial vem sofrendo mudanças radicais nos últimos anos com o advento da inovação tecnológica, globalização dos mercados, exigência de qualidade, menores prazos de entrega e níveis de customização. Pequenas e médias empresas buscam novas vias de desenvolvimento capazes de lidar com a complexidade crescente do ambiente de negócios e garantir acesso a novas fontes de vantagem competitiva (Esposito et al., 2009). 
Oliveira (2008) afirma que a busca da competitividade no atendimento às necessidades do cliente final tem gerado a necessidade de melhor organizar os processos de produção e logística, tornando mais ágil toda a cadeia do processo produtivo. A busca por um processo produtivo bem estruturado, com baixos níveis de estoques intermediários, maior produtividade e menores prazos de entrega, tem sido o principal desafio imposto às empresas. O SEBRAE (2017) traz alguns dados importantes acerca da panificação no Brasil em 2016, ressaltando a importância deste setor produtivo:

- a panificação está entre os seis maiores segmentos da indústria do Brasil, com participação de 36\% na indústria de produtos alimentares;

- aproximadamente 63,2 mil panificadores fazem parte do mercado de panificação no Brasil, dessas, 60 mil são micro e pequenas empresas $(95 \%)$;

- o setor gerou mais de 700 mil empregos diretos, sendo 245 mil (35\%) envolvidos diretamente na produção;

- $76 \%$ dos brasileiros consomem pão no café da manhã e $98 \%$ consomem produtos panificados.

O SEBRAE (2017) aponta ainda algumas dificuldades enfrentadas pelo setor. Dentre elas, destacam-se a baixa produtividade e a concorrência predatória (o maior contra o menor) e desigual.

A Associação Brasileira da Indústria de Panificação e Confeitaria (ABIP) (ABIP, 2020) destaca que em 2019, o pão francês ainda é o principal produto das padarias ( $45 \%$ de todas as padarias), a despeito de todas as inovações e diversificação do mix de produtos e serviços. Contudo, é possível perceber uma queda no volume de pão francês consumido pelos clientes das padarias tradicionais, apesar de o volume de farinha destinada para a fabricação de pão ter aumentado. A razão desta queda foi deduzida de que os consumidores passaram a buscar o pão em outros canais de venda que não apenas as padarias, refletindo a concorrência acirrada dentro do segmento (ABIP, 2020).

A ABIP (2020) trouxe ainda a informação de que muitas empresas tradicionais ainda não se atentaram para a necessidade de modernização de seus modelos, contribuindo para quedas em suas produções. Mas as empresas que buscam se atualizar, incorporar novos serviços, qualidade, frescor e diferenciação em seus produtos conseguiram permanecer no mercado.

As padarias brasileiras precisam reavaliar suas produtividades, layout, receitas, qualidade de matéria-prima e processos. O momento é de adequar com novas tecnologias, inovações, qualidade, criatividade e marketing. Inovação e renovação são boas dicas para as padarias (ABIP, 2020).

Pergher, Vaccaro e Pradella (2013) realizaram um estudo de caso em uma panificadora envolvendo diversos produtos, com a finalidade de determinar sua capacidade produtiva. Cenários hipotéticos de otimização no Arena foram sugeridos e os principais resultados obtidos se referem à adequação da capacidade dos equipamentos para atendimento da demanda. Um rearranjo na utilização dos equipamentos no modelo promoveu um aumento de até $100 \%$ na produtividade de alguns dos produtos.

Hecker, Hussein e Becker (2010) criaram um modelo de simulação analisando dez diferentes produtos de uma pequena panificadora em Estugarda, Alemanha. Os autores identificaram alguns períodos de baixa utilização de alguns dos maquinários, e tempos de ociosidade excessivos de funcionários. Após processos iterativos de alternativas de simulação, um modelo otimizado foi obtido com uma reorganização dos tempos de início dos processos e uma melhor utilização dos fornos (vários produtos de uma só vez). Os funcionários teriam turnos fixos planejados de descanso, diminuindo a ociosidade em até 55 minutos, resultando em uma possível redução de até $19 \%$ de gastos com salário dos mesmos.

Wachholz (2013) estudou especificamente o processo de produção de pão francês em uma padaria em Matelândia/PR. Após simulação de modelo no ARENA, chegou-se a duas propostas de otimização: uma com pouco investimento, realocando um funcionário de outro setor para a máquina modeladora somente em seu horário de uso em determinada etapa do processo; a outra com um investimento maior, através da criação de uma linha específica para o produto e aquisição de novos funcionários, resultando em tempos de produção ainda menores (cerca de 24\%). 
Santos et al. (2014) criaram um modelo no software livre brasileiro URURAU do processo produtivo de cucas, de uma panificadora em Medianeira/PR. O gargalo do sistema foi identificado no processo de crescimento da massa, sendo sugerido a aquisição de uma estufa para reduzir o tempo de crescimento e auxiliar no controle de temperatura e umidade, resultando em uma economia em torno de $24 \%$ do tempo de produção.

Rezende et al. (2010) utilizaram o ARENA para avaliar os tempos médios de espera em filas de atendimento de uma panificadora em Goiânia/GO. Cenários de simulação foram testados e chegou-se à conclusão que a aquisição de mais três funcionários (atualmente são sete) eliminaria o gargalo do sistema e reduziria o tempo médio de espera em 89,2\%.

Iannoni e Morabito (2002) desenvolveram um modelo complexo do sistema logístico de recepção de cana-de-açúcar da Usina São Martinho, em Pradópolis (SP), considerada a maior processadora deste produto do mundo. O processo envolve vários tipos de caminhões e diversas moendas para o processamento do produto. Uma potencial proposta de otimização foi identificada envolvendo uma melhor distribuição do atendimento dos veículos, resultando em uma redução de 13,5\% na quantidade média de cana em espera e aumento percentual de $1,1 \%$ na quantidade de cana moída.

Recentemente foi publicado um trabalho que cria um modelo, também em Arena, de um posto de lavação da cidade de Teófilo Otoni / MG (Lacerda et al., 2021). Neste trabalho, foi possível identificar alguns gargalos no sistema, além de propor melhorias de até $45,31 \%$ no tempo de execução de um dos processos, através da reorganização dos servidores na empresa. Esta alteração tem o potencial de gerar um aumento significativo na produtividade da execução do serviço.

De posse dos dados referentes aos processos produtivos coletados in loco, um modelo computacional foi criado, a fim de identificar os gargalos no sistema e apresentar possíveis otimizações para a empresa, como por exemplo, realocação de funcionários, posicionamento de máquinas, redução de filas de tempo de espera nas mesmas ou aquisição de máquinas mais eficientes.

\section{Metodologia}

A pesquisa foi dividida nas seguintes etapas representadas pela Figura 1. Este fluxo foi inspirado e segue os passos recomendados por Freitas Filho (2001), em seu livro Introdução à Modelagem e Simulação de Sistemas.

Figura 1 - Etapas de um modelo de simulação.

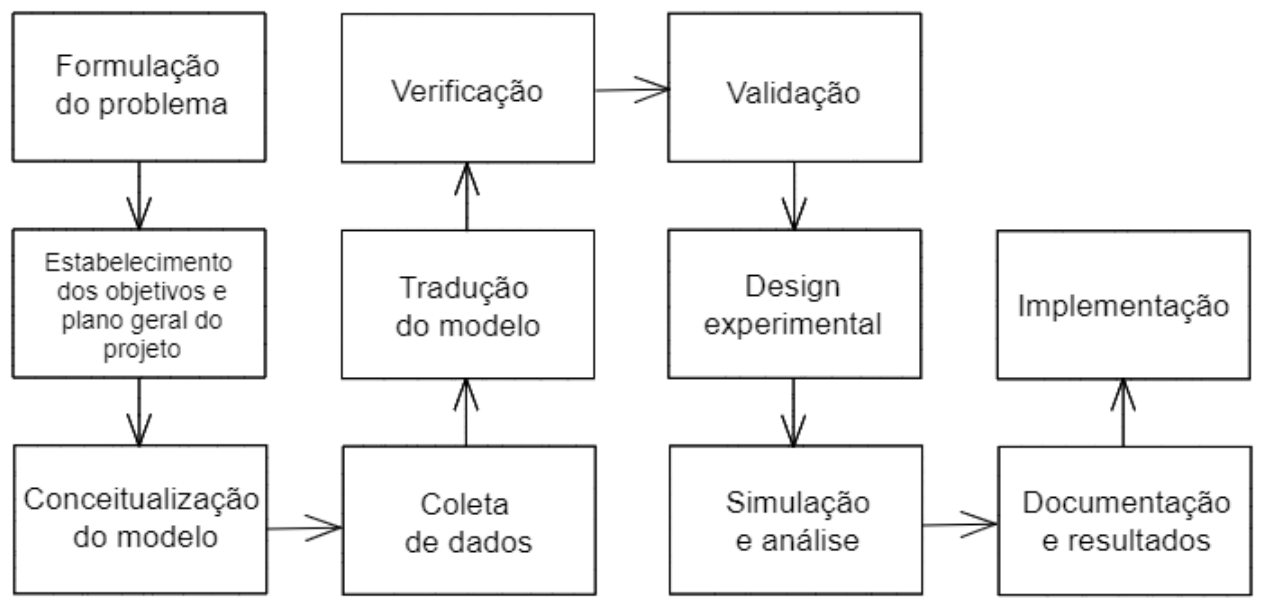

Fonte: Banks (2005). Adaptado.

Este fluxo foi inspirado e segue os passos recomendados por Freitas Filho (2001) em seu livro Introdução à Modelagem e Simulação de Sistemas. Buscou-se identificar os processos produtivos envolvidos na produção de uma padaria, escolher um 
desses processos como objeto de estudo e a partir do uso de simulação computacional propor, caso haja, melhorias e otimizações para a sua maximização produtiva. A padaria estudada encontra-se no município de Itambacuri/MG, Brasil.

Silva e Menezes (2005) em sua metodologia, pressupõem que a pesquisa científica pode ser classificada quanto a quatro abordagens diferentes, sendo estas: natureza, objetivos, forma de abordar o problema e procedimentos técnicos. Este modelo, como representado na Figura 2, foi utilizado para a classificação deste trabalho de pesquisa.

Figura 2 - Classificação de pesquisas científicas.

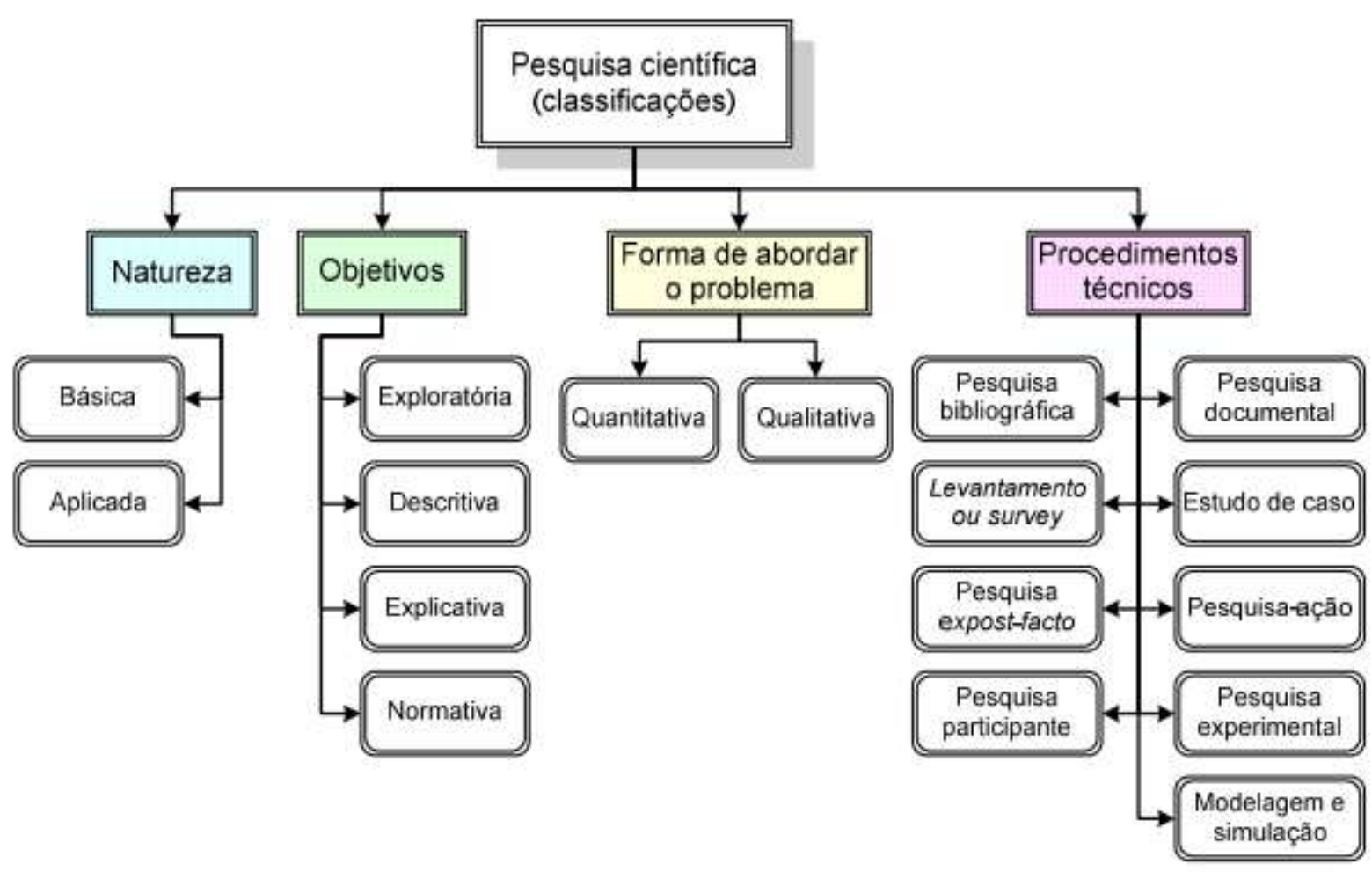

Fonte: Silva e Menezes (2005).

Quanto à natureza, o projeto se enquadra como pesquisa aplicada, que segundo Silva e Menezes (2005), objetiva gerar conhecimentos para aplicação prática prevista. Do ponto de vista da forma de abordagem, a pesquisa é quantitativa pois vem traduzir em números opiniões e informações para classificá-las e analisá-las por uso de recursos e de técnicas estatísticas (Silva \& Menezes, 2005). A respeito de seus objetivos, pode ser considerada como pesquisa descritiva que, segundo Gil et al. (2002), visa descrever as características de determinado fenômeno, envolvendo técnicas padronizadas de coleta de dados e estabelecendo relações entre variáveis. O projeto envolve também a pesquisa experimental, através da modelagem e simulação; e pesquisa bibliográfica, por utilizar-se de conhecimentos a partir de material já publicado de livros, artigos e periódicos, para a comparação com trabalhos similares e o aprendizado do uso da ferramenta (Gil et al., 2002).

A pesquisa foi dividida nas seguintes etapas representadas pela Figura 3. 
Figura 3 - Etapas da pesquisa.

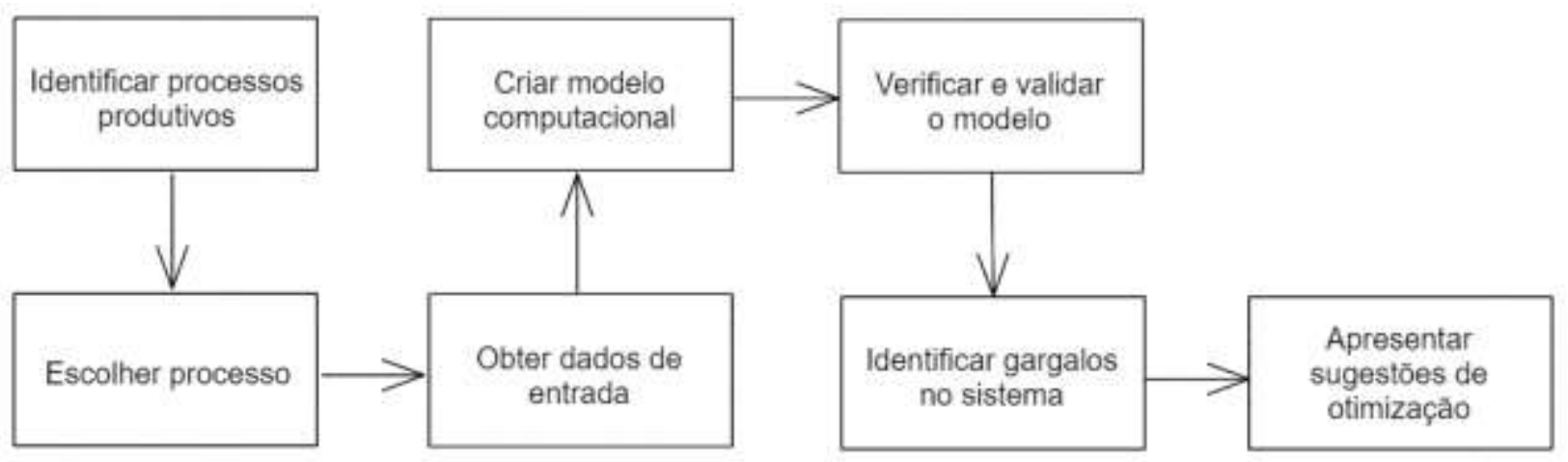

Fonte: Autores.

Este fluxo foi inspirado e segue os passos recomendados por Freitas Filho (2001), em seu livro Introdução à Modelagem e Simulação de Sistemas. Buscou-se identificar os processos produtivos envolvidos na produção de uma padaria, escolher um desses processos como objeto de estudo e a partir do uso de simulação computacional propor, caso haja, melhorias e otimizações para a sua maximização produtiva. A padaria estudada encontra-se no município de Itambacuri/MG, Brasil.

Inicialmente, foi realizado um estudo abrangente identificando os variados processos produtivos do estabelecimento e seus produtos fabricados. Dentre alguns destes produtos, podem-se citar: pão francês, pão sovado, pão de doce, rosca de leite, rosca rainha, dentre outros. Alguns desses produtos possuem massa própria (como por exemplo o pão francês). Outros são derivados de uma mesma massa. Diversos trabalhos semelhantes a este já tratam o pão francês como objeto de estudo. Logo este estudo visou buscar uma massa diferente e que possui um pouco mais de complexidade em seus processos.

Este período de coleta de dados dos variados processos produtivos da padaria levou cerca de 3 semanas para ser realizado, entre os dias 27/07/2020 a 14/08/2020. Após este período, buscou-se focar em uma massa específica, passando pelos processos produtivos de todos os produtos gerados. O objeto de estudo escolhido foi a massa de rosca. Esta massa gera outros 5 produtos: rosca de leite, rosca amanteigada, rosca média, pão de leite e pão tatu. Ela é produzida uma vez ao dia, na parte da manhã.

Escolhendo-se o produto a ser estudado, identificou-se o horário e os dias em que é produzido. Com isso as visitas para coletas de dados foram feitas de segunda à sexta, às 8:00h da manhã. Este período durou cerca de 3 meses, de 17/08/2020 a 20/11/2020. Durante esse período foram coletados dados referentes a cada processo de todos os produtos gerados pela massa. Para cada processo é identificado o tempo de operação (do padeira ou da máquina dependendo do processo). Estas medidas de tempo foram cronometradas com o auxílio de um smartphone Android. As medidas foram armazenadas no momento de coleta, para posterior tratamento estatístico e criação de modelo computacional.

São separados os processos determinísticos, que possuem tempos de operação fixos, dos processos estocásticos, que são aleatórios e dependem de distribuições de probabilidades (Freitas Filho, 2001). Um exemplo de processo determinístico é o tempo de assamento de massa no forno; um exemplo de processo estocástico é qualquer processo manual realizado pelo funcionário. Para os processos estocásticos, foram obtidos os tamanhos de amostras para que tenham a maior representatividade possível em seu próprio universo. Estes são calculados pela seguinte equação (Freitas Filho, 2001):

$$
n=\frac{Z^{2} \sigma^{2}}{E_{0}{ }^{2}}
$$

Tal que:

- $n$ : tamanho da amostra;

- $Z$ : valor da normal padronizada para um nível de confiança de 95\%; 
- $\sigma$ : desvio padrão amostral em torno da média;

- $E_{0}$ : erro amostral tolerável associado à média. Foi adotado em 10\%. Primeiramente foi realizada uma coleta inicial de alguns dados, entre 10 a 50 amostras, dependendo do processo. É então calculada a média e o desvio padrão, sendo este último utilizado na formulação acima para encontrar um novo valor para tamanho de amostra. Se este novo valor for inferior, então não se necessita de mais coletas para este processo. Caso contrário, volta-se ao campo de trabalho e são coletadas novas amostras. Novamente utiliza-se a formulação e esse processo iterativo é reaplicado até que se obtenha um tamanho de amostras significativo para o processo estudado (Freitas Filho, 2001). Isto é replicado em todos os processos estocásticos que participam do modelo computacional.

Para que a simulação seja executada, é preciso definir algumas configurações de simulação. O número de replicações do modelo é um destes parâmetros. Ele indica quantas vezes o modelo será executado, com a finalidade de se obter uma maior confiabilidade nos resultados obtidos, elevando também o tempo de duração da simulação. No caso de mais de uma simulação, os resultados são obtidos com as médias de todas as simulações realizadas.

O período de aquecimento é um parâmetro utilizado quando se deseja modelar um sistema complexo em que se pretende assegurar de que, no ponto de início de coleta de estatísticas, todo o sistema está em pleno funcionamento (Prado, 2010). A duração da simulação é configurada, especificando-se a unidade de tempo trabalhada. O tempo de simulação pode ser configurado como ilimitado, porém neste caso geralmente é indicado um critério de parada, por meio de uma expressão lógica ou limitação de entidades que entram ou saem do sistema.

\section{Resultados e Discussão}

De acordo com Freitas Filho (2001), na medida em que se têm os dados necessários e disponíveis, se passa para o tratamento destes dados. O processo de tratamento de dados inicia pelo desenvolvimento da distribuição de frequência dos dados seguida da elaboração de um histograma para posterior identificação da melhor distribuição de probabilidades que se adeque ao comportamento observado.

O Arena possui o módulo Input Analyzer que realiza de forma automática este procedimento e permite ao usuário escolher a distribuição de probabilidades a ser utilizada. Junto a cada distribuição, o programa realiza testes de aderência de Quiquadrado e Kolmogorov Smirnov.

O objetivo dos testes de aderência é verificar se os dados de uma amostra aderem a uma determinada distribuição teórica de probabilidades (Normal, Exponencial, Triangular, Beta, Gama, etc.) (Freitas Filho, 2001).

Neste trabalho foi utilizado o teste de Qui-quadrado, através do Input Analyzer. Segundo Forbes et al. (2011), quando um conjunto de dados é representado por um modelo teórico, o teste de aderência de Qui-quadrado é comumente utilizado para testar a qualidade do ajuste entre os pontos de dados observados e os valores previstos pelo modelo, desde que as diferenças sejam normalmente distribuídas. Duas hipóteses são levantadas (Freitas Filho, 2001):

- $H_{0}$ : a variável aleatória X segue a distribuição sob hipótese com os parâmetros estimados

- $H_{I}$ : a variável aleatória $\mathrm{X}$ não segue a distribuição sob hipótese com os parâmetros estimados

O valor do nível de significância utilizado foi de 5\%. Caso o valor-p encontrado para a cada distribuição em estudo seja maior que $\alpha$, aceita-se $H_{0}$, a distribuição pode ser utilizada. Caso contrário, rejeita-se $H_{0}$.

Junto ao teste de aderência, o Input Analyzer promove o cálculo do Square Error. Esse valor é obtido a partir do 
somatório das diferenças entre as frequências relativas observadas e as frequências relativas esperadas, tomadas ao quadrado. Quanto menor o valor melhor a aderência da curva de distribuição, podendo ser utilizado para comparar diferentes distribuições de probabilidade (Freitas Filho, 2001).

A verificação consiste em avaliar se o modelo é consistente em sua lógica, sem apresentar erros de simulação. A validação é mensurar se o modelo computacional é aplicável ao mundo real, se ambos possuem resultados compatíveis. Para a verificação, Freitas Filho (2001) sugere que a simulação seja testada utilizando modelos determinísticos. Esta abordagem é importante pois a variabilidade das respostas do programa devido ao comportamento das variáveis aleatórias pode dificultar a interpretação. Assim, para fins de verificação, foram atribuídos valores determinísticos a estas variáveis. Com isto, fica mais simples a previsão de respostas do sistema e, como consequência, a verificação e busca de erros nos vários módulos do programa. $\mathrm{Na}$ validação no modelo, foi utilizado o teste-t para amostras independentes na comparação entre resultados do modelo simulado e do processo real, tendo como variável resposta o tempo total de execução de todo o processo produtivo. Foi utilizado um nível de significância $\alpha$ de 5\%. Freitas Filho (2001) reforça que o teste-t é útil quando se tem a existência do sistema real, como é o caso deste trabalho. As hipóteses do teste são as seguintes:

- $H_{0}$ : as médias da variável aleatória $\mathrm{X}$ do modelo e do sistema real são iguais.

- $H_{I}$ : as médias da variável aleatória $\mathrm{X}$ do modelo e do sistema real são diferentes. Caso o valor-p seja maior que $\alpha$, aceita-se $H_{0}$ e o modelo pode ser validado segundo esse critério. Caso o valor-p seja menor que $\alpha$, rejeita-se $H_{0}$, e o modelo deve ser revisto.

Para fins de análise de resultados, foram analisados os tempos gastos no processamento de cada produto estudado, o tamanho de filas no sistema e o índice de utilização de cada maquinário. Segundo Freitas Filho (2001), a determinação de intervalos de confiança para as variáveis de interesse que medem o desempenho do sistema é um componente fundamental no processo de análise de resultados. Como em qualquer processo de estimação, a preocupação em minimizar os erros deve ser constante. Para amostras de tamanho $n \leq 30$, o semi-intervalo $h$ é calculado por Freitas Filho (2001):

$$
h=t_{n-1,1-\frac{\alpha}{2}} \frac{S}{\sqrt{n}}
$$

Em que o valor $t_{n-1,1-\frac{\alpha}{2}}$ é o valor tabulado de $t, \frac{s}{\sqrt{n}}$ o erro padrão e $\alpha$ o nível de confiança em todo o intervalo. No caso de amostras com $n>30, h$ é calculado por (Freitas Filho, 2001):

$$
h=z \frac{s}{\sqrt{n}}
$$

Em que $z$ é o valor da variável normal padronizada para o nível de confiança desejado. Em se tratando de simulação, pode-se elevar o nível de confiança com o aumento do número de replicações do modelo. Uma estimativa do número de replicações para a simulação pode ser obtida com a seguinte equação (Freitas Filho, 2001):

$$
n^{*}=n\left(\frac{h}{h^{*}}\right)^{2}
$$

Tal que:

- $n^{*}=$ nova estimativa para $\mathrm{n}$.

- $n$ = número de replicações já realizadas.

- $h$ = semi-intervalo de confiança já obtido.

- $h^{*}=$ semi-intervalo de confiança desejado. É um processo iterativo, até que se obtenha o número de replicações para o semi intervalo de confiança desejado. Segundo Freitas Filho (2001), é comum que se busquem intervalos de confiança para os quais 
o valor de h seja menor ou igual a 10\% da média amostral. Assim, este valor foi utilizado neste trabalho, juntamente com um chute inicial $n$ de 20 replicações para o modelo computacional.

A comparação de alternativas de projetos costuma ser uma atividade comum entre usuários de modelagem e simulação de sistemas. Alternativas ao modelo podem compreender desde uma mudança em um parâmetro até uma lógica diferenciada que reflita uma variação fundamental no funcionamento do sistema (Freitas Filho, 2001). No presente trabalho, para verificar se as diferenças entre os resultados das propostas de otimização e o modelo foram ou não significativas, foi realizado o teste t para amostras independentes, já descrito anteriormente. As alternativas de otimização foram propostas tendo como base a variação do número de padeiros ativos no sistema. Esta decisão foi escolhida por ser uma alternativa viável e mais próxima do cenário real de otimização. Os mesmos parâmetros foram reproduzidos em simulações diferentes, alternando-se o número de padeiros de 1 a 10. Sabe-se que tal magnitude no número de operários é irreal e não se aplica em uma padaria deste tamanho, porém a comparação é válida para fins de estudo de logística. A comparação de resultados entre simulações diferentes se deu basicamente pela comparação percentual entre as médias dos tempos totais de execução do processo como um todo, ou seja, o tempo total de simulação. Foram incorporados ao modelo tanto as médias de tempo de processamento de cada processo, quanto as médias dos resultados de todas as replicações do modelo (20 replicações).

A massa estudada (massa de rosca) é responsável por gerar cinco pães finais: rosca de leite, rosca amanteigada, rosca média, pão tatu e pão de leite. A Figura 4 ilustra essa primeira etapa do modelo.

Figura 4 - Processo produtivo parte 1.

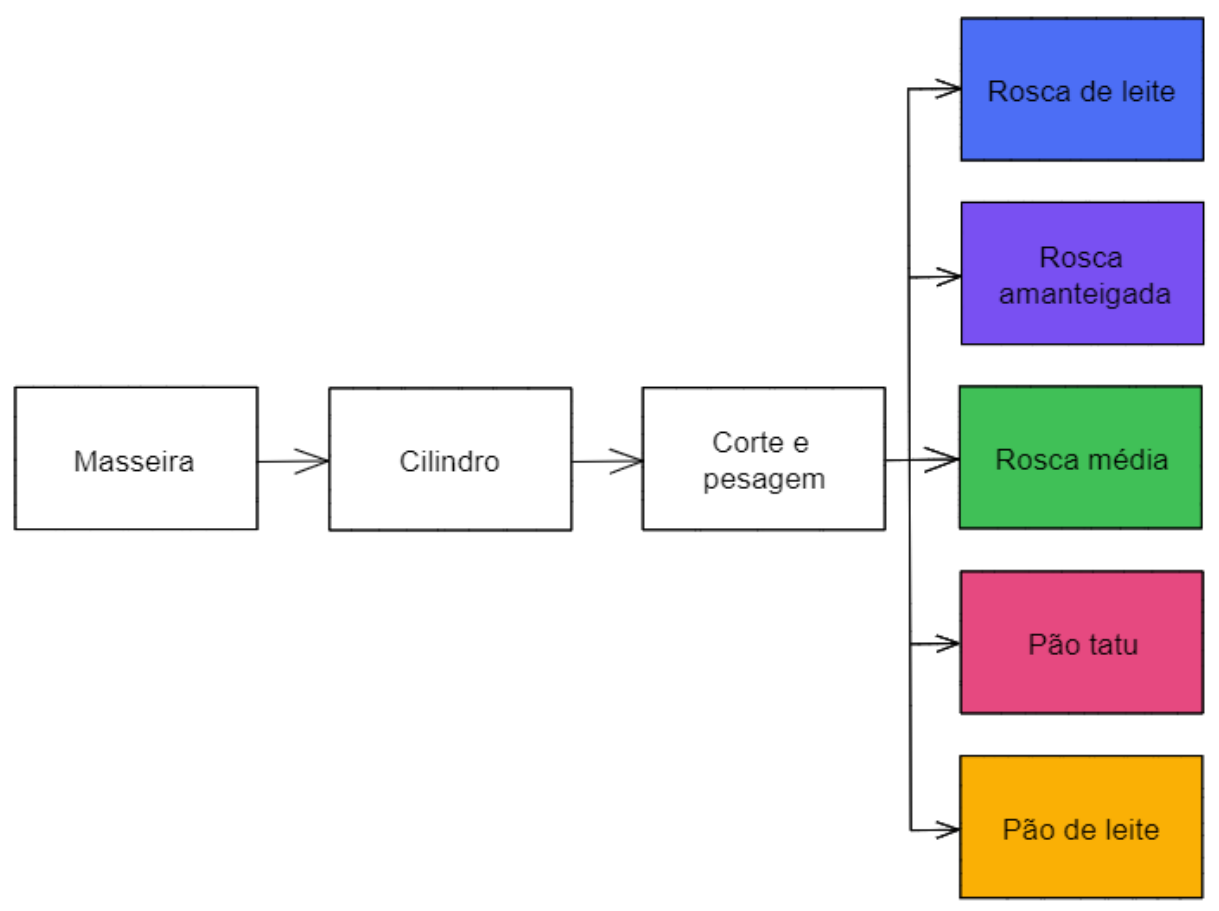

Fonte: Autores.

A massa inicial é preparada na masseira e no cilindro, e depois é dividida pelo processo de corte e pesagem. Com exceção da masseira que é um processo determinístico, todos os outros processos envolvidos neste modelo de simulação são estocásticos. A Figura 5 continua com o fluxo para cada produto. 
Figura 5 - Processo produtivo parte 2.
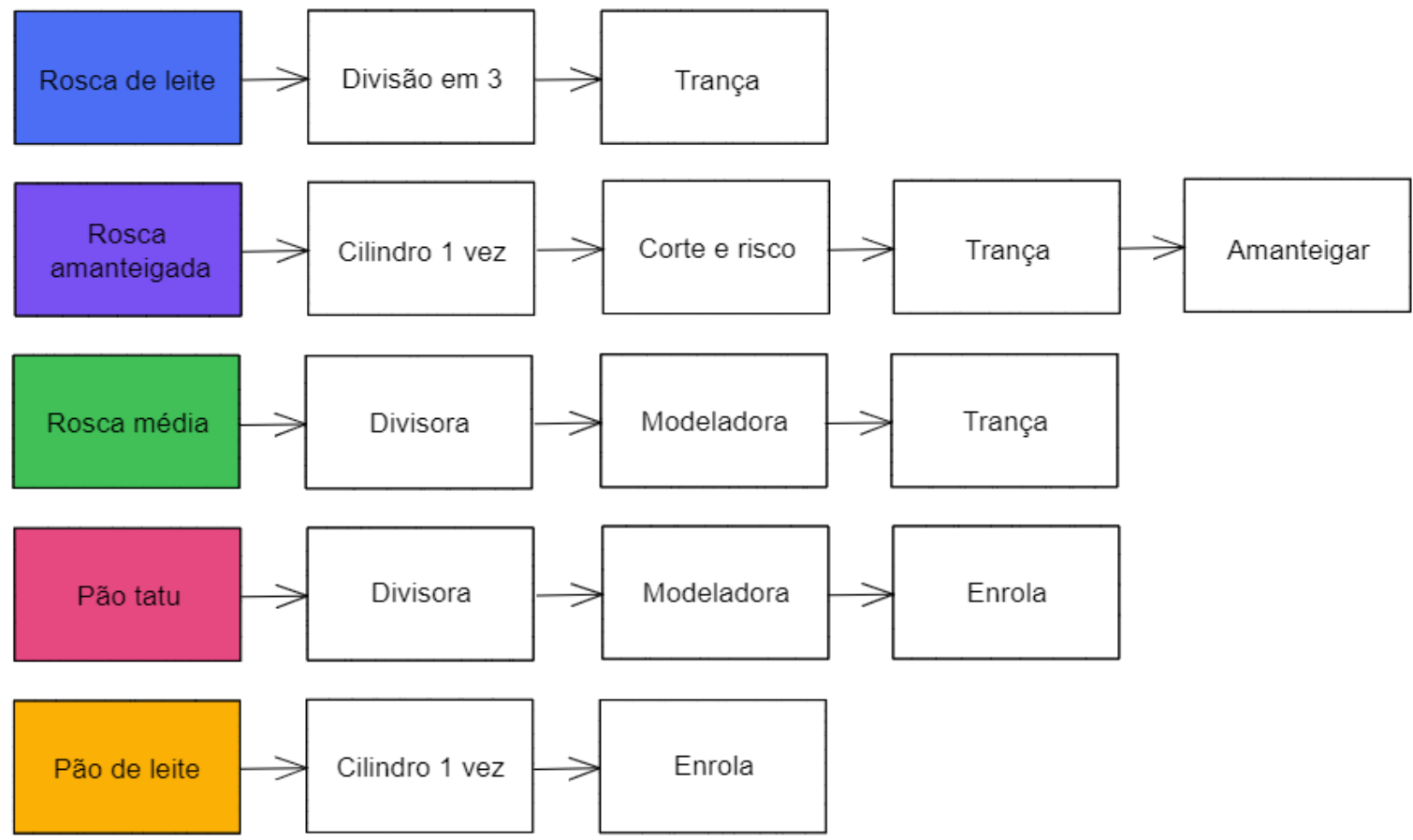

Fonte: Autores.

Cada produto passa por processos em série diferentes, porém compartilhados entre si. Alguns deles passam novamente pelo cilindro (porém 1 só vez ou passada) para preparar a massa para os próximos processos.

A rosca média e o pão tatu são produtos de tamanhos menores, e por isso a massa geradora passa por uma divisora e modeladora (auxilia no processo de enrolamento ou trança).

A rosca de leite, a rosca amanteigada e o pão de leite são produtos maiores. A peça inteira é processada sem divisão adicional. A Figura 6 ilustra alguns dos produtos logo depois de preparados.

Figura 6 - Fotografias de maquinários - masseira e cilindro.

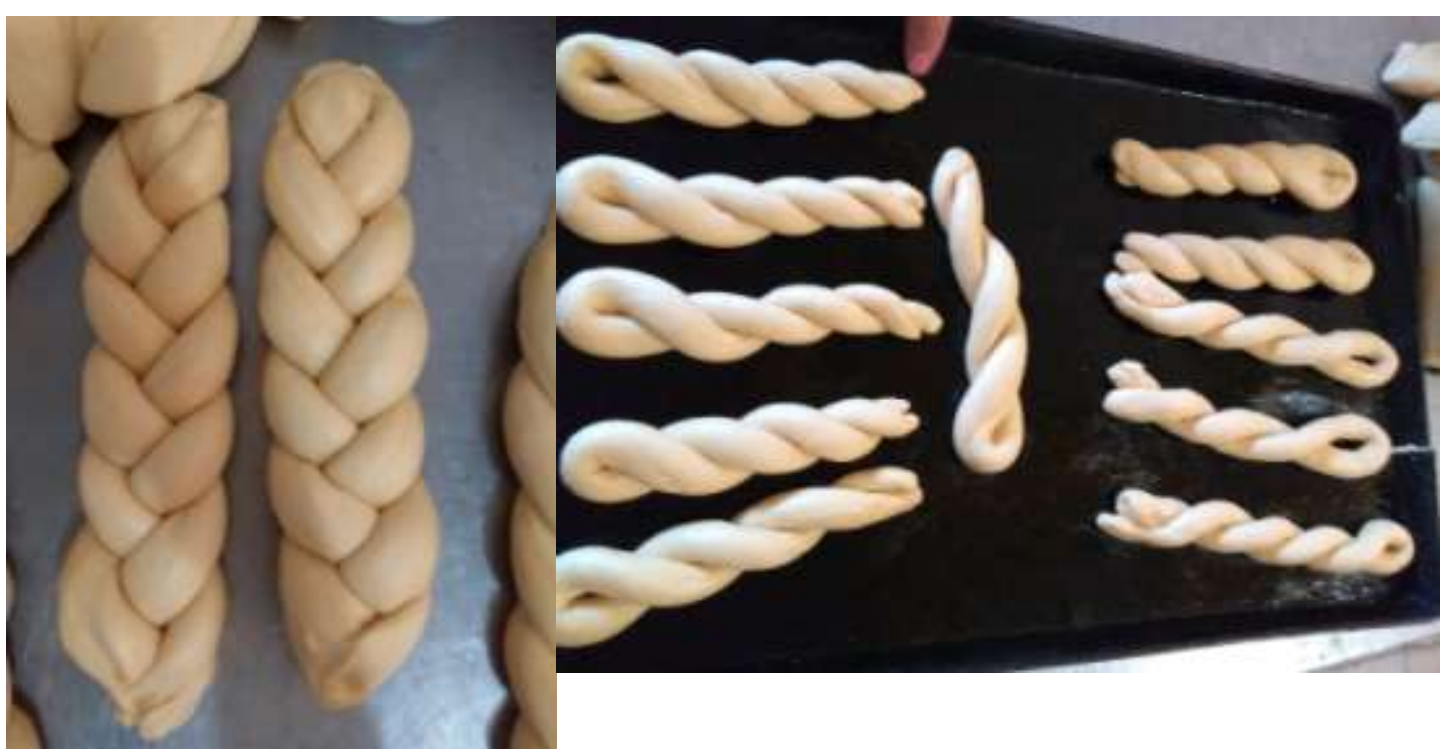

(a)

(b)

Fonte: Autores. 
O modelo estudado neste trabalho analisa os processos envolvidos do início da mistura dos ingredientes na masseira até a disposição final em assadeiras. Portanto, desconsidera-se os tempos de pesagem inicial de ingredientes, tempos de estufa e tempos de forno. Processos envolvidos na produção:

- Masseira: processo determinístico, com tempo fixo da máquina de mistura. Cada ingrediente é colocado na masseira e são misturados durante um tempo fixo, como mostrado na Figura 7 (a).

- Cilindro: processo envolvendo contato contínuo de padeiro, utilizado para sovar a massa, como mostrado na Figura 7 (b). A massa é dividida em 2 partes e sovadas de forma separada.

- Corte e pesagem: a massa é dividida em partes menores, dependendo do pão a ser produzido. Algumas dessas partes se tornam pães inteiros (no caso das roscas de leite, amanteigada e pão de leite), outras são divididas em outras porções menores posteriormente na divisora. 11 massas são resultantes deste processo.

Figura 7 - Fotografias de maquinários - masseira e cilindro.

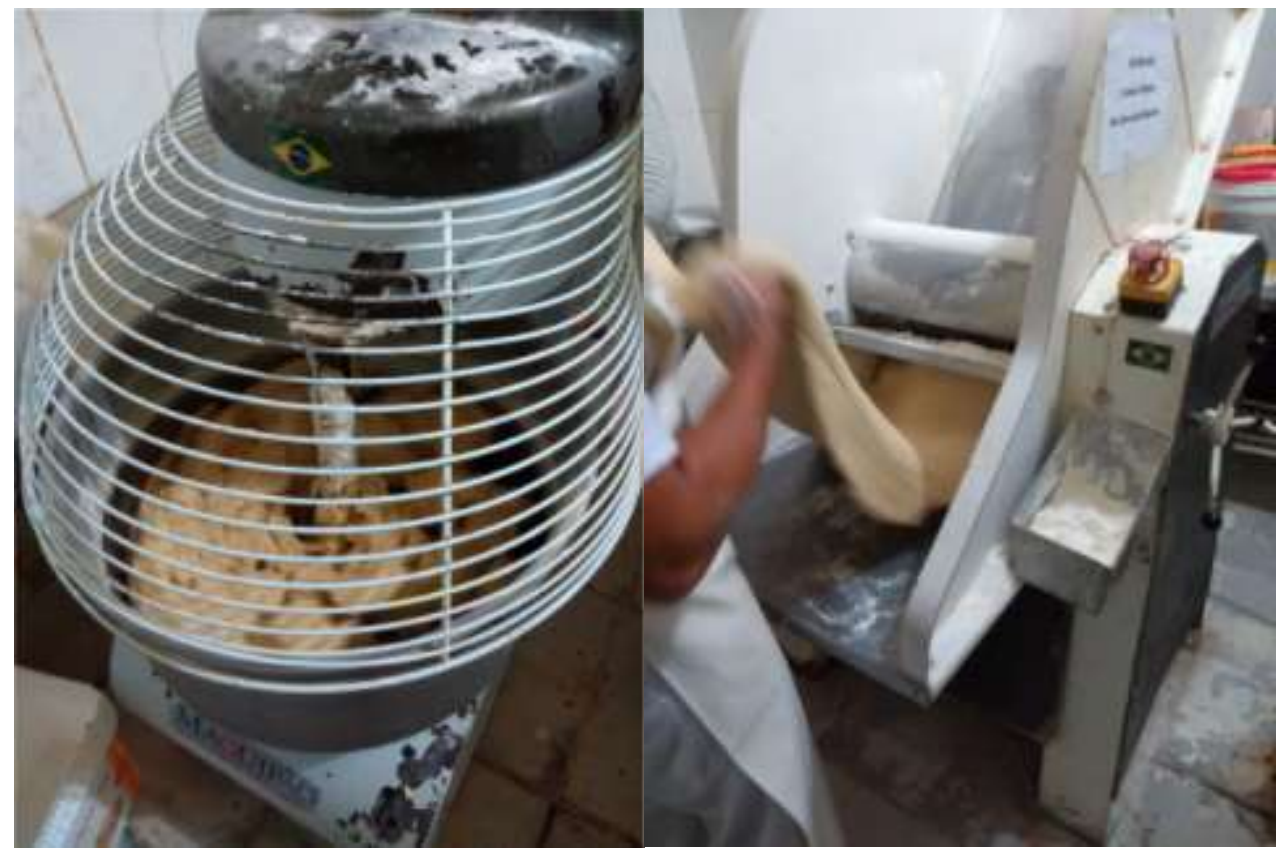

(a)

(b)

Fonte: Autores.

- Divisora: as massas que necessitam ser divididas em porções menores passam por este maquinário, identificado pela Figura 8 (a), em que cada uma é dividida em 30 partes.

- Modeladora: responsável por modelar as massas de pão tatu e rosca média, identificado pela Figura 8 (b).

- Enrolamento, trança e passagem de manteiga: processos manuais executados na mesa de trabalho. 
Figura 8 - Fotografias de maquinários - divisora e modeladora.

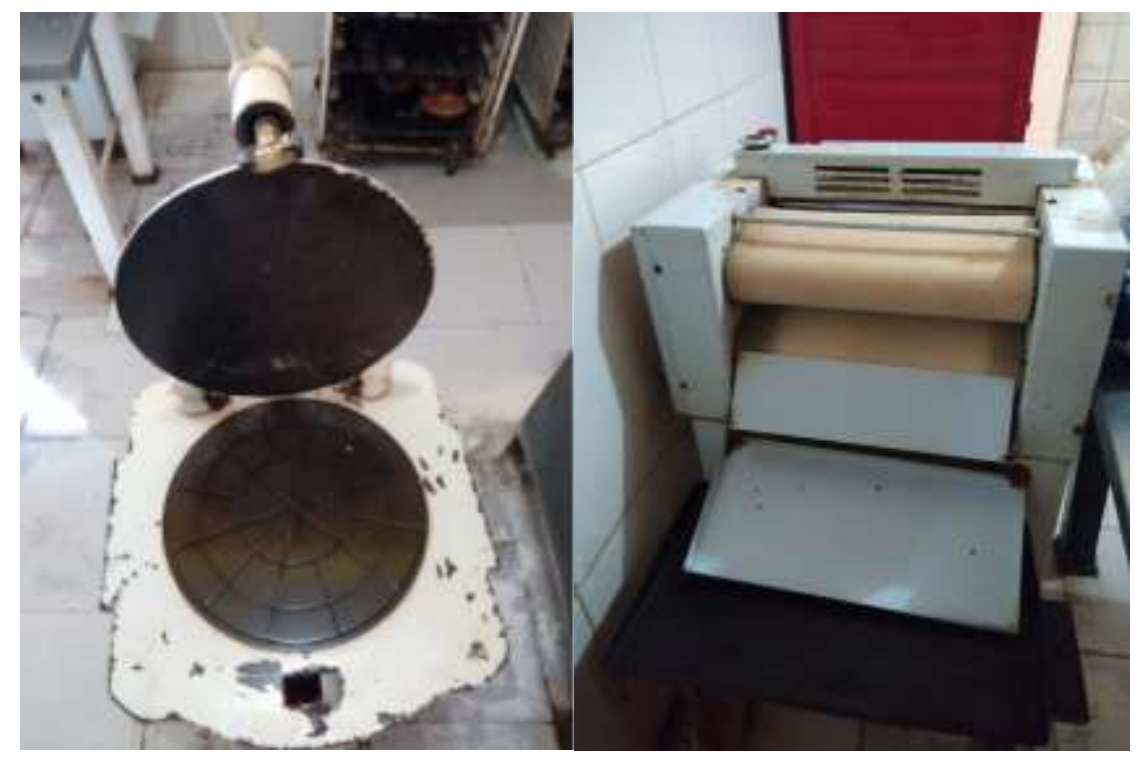

(a)

(b)

Fonte: Autores.

O modelo produzido obteve como resultado final uma média de 40,77 minutos dentre 20 simulações. Este valor foi validado com a situação real com média de 39,80 minutos. Vale ressaltar que o modelo foi construído com as coletas individuais de cada processo envolvido nos produtos resultados da Massa de Rosca. O funcionamento total do sistema como um todo concatenou na obtenção deste resultado final.

A maioria dos processos apresentou fila média com menos de uma unidade, com exceção de quatro processos: modeladora (7,95 unidades) e processo de enrolamento (10,05 unidades) para rosca média, modeladora (2,95 unidades) para pão tatu e enrolar pão tatu (6.19 unidades).

Estes são os maiores gargalos do sistema, principalmente o processo de enrolamento da rosca média. Estes processos podem estar atrasando toda a produção como um todo. São pontos passíveis de otimização.Para efeitos de comparação e busca de otimização, foram realizadas outras simulações de 20 replicações cada, variando-se o número de padeiros em exercício na produção estudada. Os resultados foram plotados no gráfico da Figura 9. 
Figura 9 - Comparação entre cenários com diferentes números de padeiros.

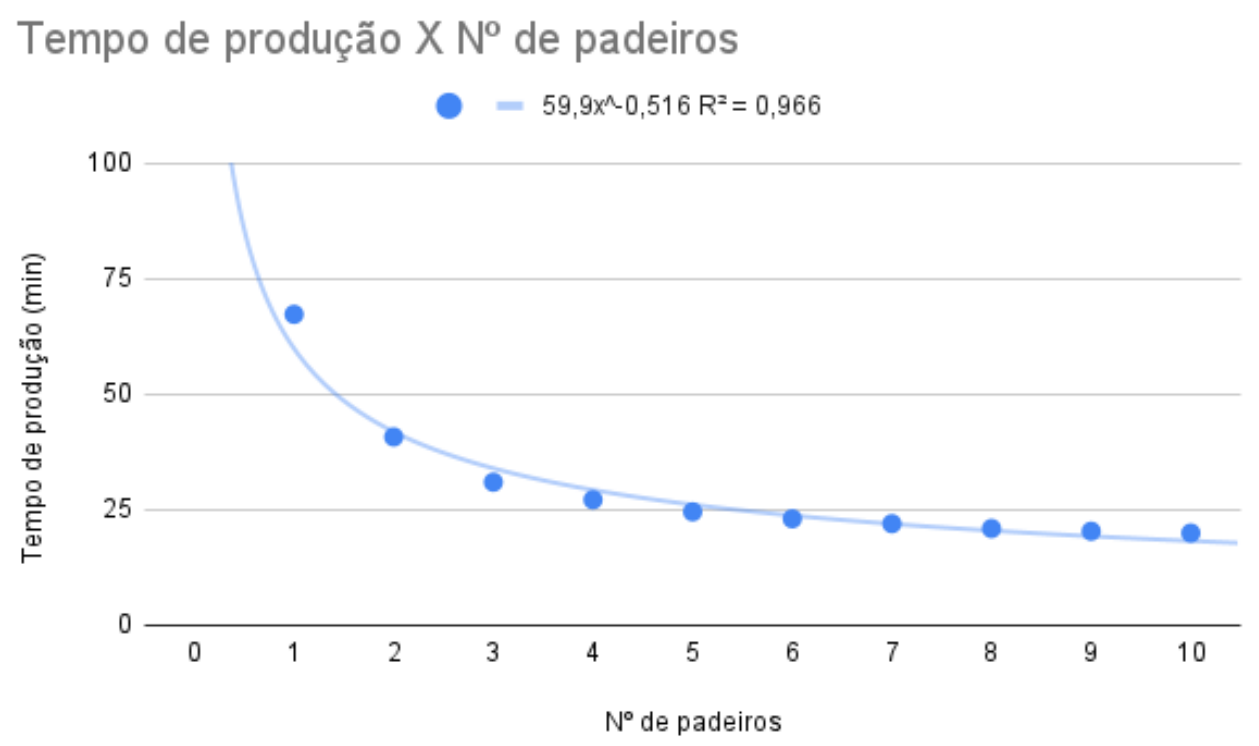

Fonte: Autores.

Não é viável economicamente, nem logisticamente, alocar estas quantidades de padeiros para a produção desta massa. Porém a comparação foi feita para se ter um entendimento do comportamento do fenômeno. Como é observado na Figura 9, existe um decaimento rápido até 3 padeiros, quando começa a atingir uma constância. Pôde-se traçar uma curva de tendência com coeficiente de determinação $R^{2}=0,966$ obedecendo a equação $y=59,9 x-0,516$.

O processo real adota a situação de utilizar 2 padeiros. Entre o uso 2 padeiros e 3 padeiros (30,98 minutos), há uma redução de $24 \%$. Esta alternativa pode vir a se tornar viável. Não necessariamente precisa-se do terceiro padeiro em todos os processos, mas principalmente nos gargalos do sistema. É uma situação de otimização que tem potencial de reduzir gastos e tempo, que pode vir a ser analisada em um estudo futuro.

\section{Considerações Finais}

A princípio, este trabalho iria realizar o estudo descrito em algumas outras padarias da região. Porém, devido à pandemia de Covid-19 entre os anos de 2020 e 2021, a logística de visita aos locais de trabalho foi prejudicada e se tornou restrita e difícil de ser realizada. Assim, o objeto de estudo deste trabalho ficou restrito à elaboração de modelo computacional para uma padaria.

Neste quesito, acredito que a pesquisa demonstra relevância no sentido em que foi encontrado um resultado positivo após a simulação. O modelo computacional foi capaz de atender a grande parte das características de uma situação real e conseguiu propor alternativas para otimização do processo produtivo.

Apesar disso, o modelo é uma simples abstração de todo o processo real. Diferentes profissionais chegarão a resultados distintos com modelos semelhantes, desde que sejam boas reproduções da realidade.

Um modelo não precisa ser extremamente complexo para ser funcional. A simplicidade de abstrações bem feitas muitas vezes agilizam o processo de modelagem. Algumas informações são supérfluas e não têm impacto significativo nos resultados.

Para trabalhos futuros, fica a proposta de aplicar este modelo de otimização e realizar novamente a coleta de dados para a sua validação em um novo modelo computacional. Esta seria a comprovação final deste trabalho, de que um modelo computacional realizou o seu propósito de promover uma melhoria em um processo produtivo real. Outra sugestão seria a realização de estudo similar com a massa de pão francês na mesma padaria, pelo fato de ser um pão comum no Brasil. Os 
resultados poderiam ser comparados com outros estudos de simulação realizados para a mesma massa.

\section{Referências}

ABIP. (2020). Indicadores de panificação e confeitaria brasileira em 2019. https://www.abip.org.br/site/wp-content/uploads/2020/02/INDICADORES-DAPANIFICA\%C3\%87\%C3\%83O-E-CONFEITARIA-EM-2019-2.pdf

Banks, J. (2005). Discrete event system simulation. Pearson Education India.

Esposito, E. et al. (2009). Virtual enterprise in SME networks. Piccola Impresa/Small business.

Forbes, C. et al. (2011). Statistical distributions. John Wiley \& Sons.

Freitas Filho, P. J. d. (2001). Introdução à modelagem e simulação de sistemas: com aplicações em Arena. Visual Books.

Gil, A. C. et al. (2002). Como elaborar projetos de pesquisa. Atlas São Paulo. 4.

Hecker, F., Hussein, W., \& Becker, T. (2010). Analysis and optimization of a bakery production line using ARENA. International Journal of Simulation Modelling, 9(4), 208-216.

Iannoni, A. P. \& Morabito, R. (2002). Análise do sistema logístico de recepção de cana-de-açúcar: um estudo de caso utilizando simulação discreta. Gestão \& produção, 9, 107-127.

Kelton, W. D. (2002). Simulation with ARENA. McGraw-hill.

Lacerda, L. P. et al. (2021). Aplicação da teoria de filas via modelagem computacional em uma empresa de lavagem de veículos. Research, Society and Development

LEAP. Industry 4.0. (2020). https://www.leapaust.com.au/industry-4-0/

Moura, M. d. C. \& Nascimento, L. M. d. (2020). Indústria 4.0 e simulação de processos produtivos: como os gêmeos digitais podem ajudar na busca da excelência operacional? https://www.excelenciaoperacional.blog.br/2018/08/31/industria-4-0-e-simulacao-de-processos-produtivos-como -os-gemeos-digitais- podemajudar-na-busca-da-excelencia-operacional/

Oliveira, C. S. d. (2008). Aplicação de técnicas de simulação em projetos de manufatura enxuta. Estudos Tecnológicos em Engenharia, 4(3), $204-217$.

Pergher, I., Vaccaro, G. L. \& Pradella, M. (2013). Aplicação da simulação computacional para determinar a capacidade produtiva do processo de produção de pães: um estudo de caso. Produto \& Produção, 14(1).

Prado, D. (2010). Usando a arena em simulação. INDG Tecnologia e Serviços Ltda.

Rezende, H. P. de et al. (2010). Avaliação dos tempos de espera em uma panificadora utilizando simulação computacional. XXX Encontro Nacional de Engenharia de Produção.

Santos, A. A. d. et al. (2014). Modelagem e análise de um processo de produção em uma pequena panificadora utilizando um simulador de código livre. Revista de Engenharia e Tecnologia, 6(1), 148.

SEBRAE. (2017). Indústria: Panificação. https://www.sebrae.com.br/Sebrae/Portal\%20Sebrae/UFs/BA/Anexos/Ind\%C3\%BAstria\%20da\%20panifica\%C3\% A7\%C3\%A3o.pdf

Silva, E. L. d. \& Menezes, E. M. (2005). Metodologia da pesquisa e elaboração de dissertação. UFSC, Florianópolis, (4a ed.), 123.

Wachholz, L. (2013). Análise e simulação do processo de produção de pão francês em uma panificadora de pequeno porte: um estudo de caso. Trabalho de Conclusão de Curso de Bacharel em Engenharia de Produção-Universidade Tecnológica Federal do Paraná. Medianeira. 